\title{
Simulations of solar magnetic dynamo action in the convection zone and tachocline
}

\author{
Matthew K. Browning ${ }^{1}$, Mark S. Miesch ${ }^{2}$, Allan Sacha Brun ${ }^{3}$ \\ and Juri Toomre ${ }^{4}$ \\ ${ }^{1}$ Astronomy Dept, University of California at Berkeley, Berkeley, CA 94720-3411, USA \\ email:matthew@astro.berkeley.edu \\ ${ }^{2}$ High Altitude Observatory, NCAR, Boulder, CO 80307-3000 \\ email:miesch@ucar.edu \\ ${ }^{3}$ DSM/DAPNIA/SAp, CEA Saclay, 91191 Gif sur Yvette, France \\ email:allan-sacha.brun@cea.fr \\ ${ }^{4}$ JILA and Dept. of Astrophysical and Planetary Sciences, U. Colorado Boulder, Boulder, \\ CO 80309-0440 \\ email:jtoomre@lcd.colorado.edu
}

\begin{abstract}
We present results from a global 3-D nonlinear simulation of magnetic dynamo action achieved by solar convection in a penetrative geometry. We include within the spherical computational domain both the bulk of the convection zone and a portion of the underlying stable layer. A tachocline of rotational shear is realized below the convection zone, where we have imposed both a hydrodynamic drag term and small thermal perturbations consistent with thermal wind balance. Thus we are capturing many of the dynamical elements thought to be essential in the operation of the global solar dynamo, including differential rotation arising from convection, magnetic pumping, and the stretching and amplification of toroidal fields within the tachocline. In the stable region, the simulation reveals that strong axisymmetric toroidal magnetic fields (about $3000 \mathrm{G}$ in strength) are realized, in contrast to the mostly fluctuating fields that predominate in the convection zone. The toroidal fields in the stable region exhibit a striking antisymmetric parity akin to that observed in sunspots, with fields in the northern hemisphere largely of the opposite sign to those in the southern hemisphere. These deep toroidal fields are accompanied by mostly dipolar mean poloidal fields, whose polarity has retained the same sense over multiple years of simulated evolution.
\end{abstract}

Keywords. Convection, turbulence, magnetic fields, MHD, Sun: magnetic fields

\section{Building Blocks of Solar Magnetism}

The most widely accepted paradigm for explaining the origins of the 22-year cycle (see Parker 1993; Charbonneau \& MacGregor 1997; Ossendrijver 2003). This model proposes that the global solar dynamo involves several conceptually distinct dynamical "building blocks," operating at different sites within and below the convection zone. These processes likely include a) magnetic field generation by intensely turbulent convection influenced by rotation within the bulk of the convective envelope, b) the pumping or transport of those fields downward into the tachocline of rotational shear near the base of that zone, c) the stretching of those fields to form strong toroidal fields, and d) the rise of such field structures due to magnetic buoyancy, leading to field loops that ascend toward the surface. Prior global simulations of the convective envelope (Brun, Miesch, \& Toomre 2004) have studied elements (a) and (b), and thereby showed that strong fluctuating magnetic fields could be generated by dynamo action within the bulk of the convective envelope, without significantly diminishing the differential rotation established there. 
Here, we report on the first 3-D global MHD simulations to incorporate element (c) as well, by allowing penetration below the convection zone into a forced tachocline of shear. We have also presented some early results of these simulations elsewhere (Browning, Miesch, Brun, \& Toomre 2006).

\section{Computational Approach}

Our 3-D nonlinear MHD simulation is intended to be a simplified description of the bulk of the solar convection zone and a portion of the underlying stable region. The spherical computational domain extends from 0.62 to $0.94 \mathrm{R}$, with $\mathrm{R}$ the solar radius, thereby encompassing most of the convection zone and part of the radiative interior. We utilize the Anelastic Spherical Harmonic (ASH) code (e.g., Miesch et al. 2000), which solves the anelastic MHD equations within a spherical shell, to examine turbulent convection and magnetism under the influence of rotation. Our calculations include spherical harmonic degrees up to $\ell=340$; in the radial direction, we adopt a stacked Chebyshev expansion with 98 collocation points. The initial stratifications of density, temperature, and pressure are consistent with a 1-D stellar model. We have softened the steep transition between the convective and stable regions, in order to avoid driving high-frequency gravity waves that we cannot readily resolve. We began the calculations here by introducing a small-amplitude seed magnetic field into a mature progenitor hydrodynamic simulation. This seed field was purely toroidal, confined to the convection zone, and contained both symmetric and antisymmetric modes. We then imposed a simulated tachocline as described presently.

In the Sun, the tachocline of rotational shear is believed to play a pivotal role in the operation of the global dynamo. We have therefore sought to impose such a region of shear in two complementary ways. First, we introduced a drag force upon motions in the stable region, with a radial profile constructed such that motions within the bulk of the convection zone are unimpeded by its effects. Without this drag force, any differential rotation established within the convection zone would fairly rapidly imprint upon the radiative interior through viscous and thermal diffusion, both of which are far more effective in our simulation than in the real Sun. Second, we have imposed small entropy variations near the base of the convective envelope in order to emulate the possible coupling between the convective envelope and the radiative interior through thermal wind balance within the tachocline. If the tachocline is in such a thermal wind balance, the strong angular velocity contrast there would imply the presence of latitudinal entropy variations, as discussed in Miesch, Brun, \& Toomre (2006) and Rempel (2005). Here, the imposed entropy contrast is associated with a small temperature contrast between the pole and equator (with the former about $6 \mathrm{~K}$ warmer near the base of the convection zone).

\section{Dynamo Action Yields Organized Magnetism}

The intricate convective flows establish differential rotation within the convection zone, with the equator rotating about $13 \%$ faster than the poles. The stably stratified interior is in nearly solid-body rotation, so there is a region of strong rotational shear near the base of the convective envelope. This simulated tachocline plays a major role in the generation of organized magnetism by dynamo action.

Dynamo action is readily realized, with small seed magnetic fields amplified greatly and sustained against Ohmic decay. Within the bulk of the convection zone, the magnetic fields are (as in BMT04) mostly fluctuating (3000 G rms), and are accompanied by 

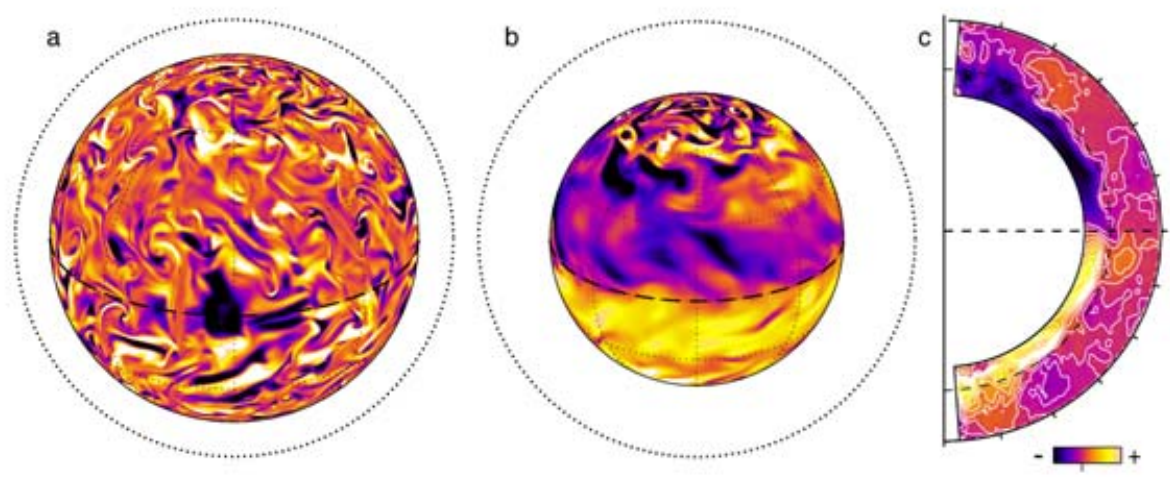

Figure 1. Toroidal magnetic fields realized in the convection zone and the underlying stable region. Shown is longitudinal field $B_{\phi}$ viewed on spherical surfaces $(a)$ at mid-depth in the convection zone $(r=0.84 R)$ and $(b)$ in the stable zone $(r=0.67 R)$. (c) Contour plot in radius and latitude of $B_{\phi}$ averaged in time and in longitude.

only weak mean poloidal and toroidal fields (with time-averaged strengths of order 300 G). The fields there possess structure on many spatial scales and exhibit no evident polarity preferences (Fig 1a). Within the stably stratified region below, however, the magnetic field has been strongly organized by the rotational shear (Fig. 1b), with the axisymmetric (mean) toroidal fields larger than the fluctuating (non-axisymmetric) fields. The time-averaged axisymmetric fields in the stable region attain strengths of order 3000 $\mathrm{G}$, or about ten times stronger than their counterparts in the convection zone. Equally strikingly, these toroidal fields exhibit antisymmetric parity, with fields in the northern hemisphere largely of opposite sign to those in the southern hemisphere. Further, the overall polarity of the axisymmetric dipole field has not flipped during the roughly 9 years of evolution we have so far simulated. This is also in sharp contrast to the fields realized in BMT04, whose polarity reversed at irregular intervals of less than 600 days.

The strong axisymmetric toroidal fields realized in the stable layer, the remarkable antisymmetric parity displayed by those fields, and the persistence of a single polarity for multiple years, are somewhat reminiscent of the highly organized magnetism that appears as sunspots at the solar surface. In future work, we plan to assess the robustness of these intriguing features.

\section{References}

Browning, M.K., Miesch, M.S., Brun, A.S., \& Toomre, J. 2006, ApJ (Letters) 648, L157

Brun, A. S., Miesch, M. S., \& Toomre, J. 2004, ApJ, 614, 1073

Charbonneau, P. \& MacGregor, K.B. 1997, ApJ, 486, 502

Parker, E. N. 1993, ApJ, 408, 707

Miesch, M.S., Brun, A.S., \& Toomre, J. 2006, ApJ, 641, 618

Ossendrijver, M. 2003, ARA\&A, 11, 287

Rempel, M. 2005, ApJ, 622, 1320 